\begin{tabular}{|c|c|c|c|}
\hline$\Omega$ & (1) & $\equiv$ & \\
\hline Rebecca S. & 'Dept of Respiratory & Rebecca S. Dickinson, & rebecca.brian@doctors.net.uk \\
\hline Dickinson', & Medicine & Hull and East Yorkshire NHS & \\
\hline Graham Robinson ${ }^{2}$, & $\begin{array}{l}{ }^{2} \text { Department of } \\
\text { Interventional Radiology, }\end{array}$ & $\begin{array}{l}\text { Trust, Administration Block, } \\
\text { Castle Hill Hospital, }\end{array}$ & \\
\hline Michael A. & Hull and East Yorkshire & Cottingham HU16 5JQ, UK & \\
\hline Greenstone $^{\top}$ & & & \\
\hline
\end{tabular}

\title{
Curable hypoxia in an octogenarian with an undiagnosed inherited condition
}

\section{Case Report}

\section{Case history}

An 84-year-old gentleman was referred to the chest clinic with low oxygen saturations on pulse oximetry. He reported breathlessness and wheeze since his retirement as a surveyor 16 years previously. His exercise tolerance was $350 \mathrm{~m}$. He had pre-existing diagnoses of chronic obstructive pulmonary disease and hypertension. He had no avian or occupational exposures, a minimal smoking history while in his teens and had not smoked since, and no history of transient ischaemic attack or cerebrovascular accident.

On examination, he was comfortable with no respiratory distress but had slight peripheral cyanosis and oxygen saturations of $89 \%$ on room air. Auscultation of his chest revealed increased vocal fremitus and crackles over the right lower lobe with occasional wheeze.

The patient was investigated using lung function testing, capillary blood gas sampling, a chest radiograph and thoracic computed tomography (CT). His haemoglobin at presentation was $16.1 \mathrm{~g} \cdot \mathrm{dL}^{-1}$.

\section{Investigations \\ Lung function}

The results of the lung function investigations performed as shown in table 1. Supine and erect spirometry demonstrated oxygen saturations of $93 \%$ when lying down and $86 \%$ on sitting. There was no significant reversibility to $2.5 \mathrm{mg}$ nebulised salbutamol.

A capillary blood gas taken on air at rest showed an oxygen tension $\left(\mathrm{PO}_{2}\right)$ of $8.0 \mathrm{kPa}$ (normal 12.0-15.0 kPa), a carbon dioxide tension $\left(\mathrm{PCO}_{2}\right)$ of $4.4 \mathrm{kPa}$ (normal 4.5-6.1 $\mathrm{kPa}$ ) and $\mathrm{pH}$ of 7.44 (normal 7.36-7.42).

\section{CT}

CT images are shown in figure 1.
Statement of Interest None declared. 
Table 1. Lung function

\begin{tabular}{lcccc} 
Parameter & Measured & Predicted & \% predicted & 50 \\
\hline FEV L & 1.64 & 3.30 & 92 & -3.27 \\
\hline FVC L & 4.11 & 4.47 & 55 & -0.60 \\
\hline FEV $/$ FVC \% & 40 & 72 & 56 & -4.55 \\
\hline TLCO & 5.42 & 9.67 & 76 & -3.01 \\
\hline KCO & 0.91 & 1.19 & 129 & 2.15
\end{tabular}

SR: standard residual (normal \pm 1.64 ); FEVı: forced expiratory volume in 1 s; FVC: forced vital capacity; TLCO: transfer factor of the lung for carbon monoxide; KCO: transfer coefficient for carbon monoxide; RV: residual volume.
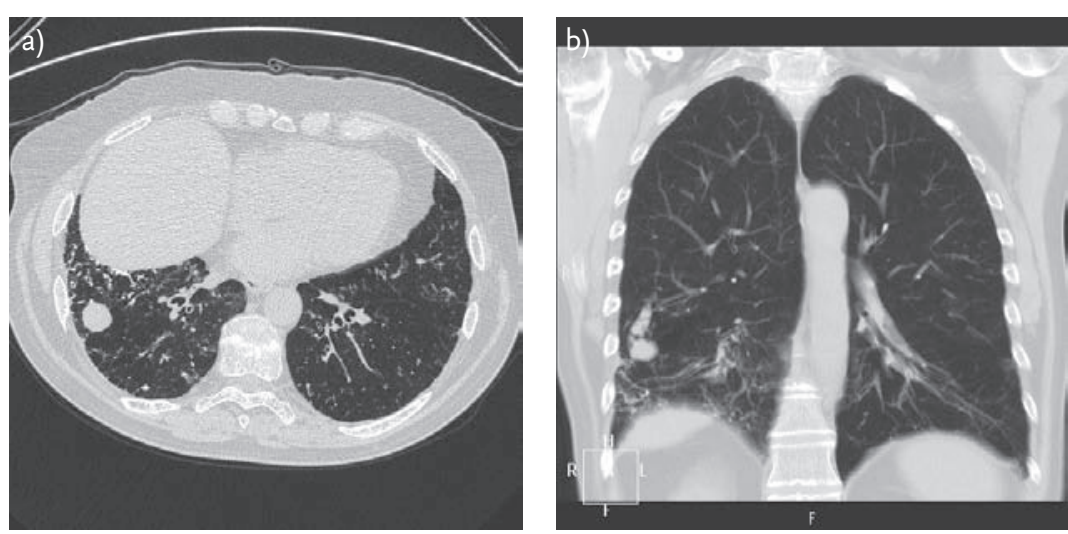

Figure 1

a) Axial and b) coronal CT slice.

\section{Task 1}

1) What is seen on the initial CT scan (fig. 1).

2) What conditions can be associated with this?

3) Can you interpret the lung function results (table 1 )? 


\section{Answer 1}

1) There is a $2-\mathrm{cm}$ vascular abnormality in the right lower lobe consistent with a pulmonary arteriovenous malformation (PAVM).

2) PAVMs are most commonly seen with hereditary haemorrhagic telangiectasia $(>90 \%)$ and post-cardiac surgery for cyanotic congenital heart disease with resultant cavopulmonary shunt. They can occur sporadically, or may very occasionally be secondary to, for example, hepatic cirrhosis or schistosomiasis.

3) The spirometry shows an obstructive picture of moderate severity. Nevertheless, the $\mathrm{FEV}_{1}$ is relatively well preserved for this degree of desaturation and it was this discrepancy that prompted the initial referral suggesting another contributing pathology. Importantly, this patient demonstrates orthodeoxia, whereby he becomes desaturated when sitting or standing from a recumbent position. This is confirmed symptomatically as his dyspnoea improved when lying flat (platypnoea, a very rare symptom for this condition).

The exact mechanism of orthodeoxia is unclear but is different to cardiac disease. One contributing factor to the orthodeoxia is that most PAVMs are at the lung bases and right-to-left shunting increases on standing because of the effects of gravity. The normal anatomical right-to-left shunt is less than $2 \%$ but is increased in patients with PAVMs. The size of the shunt influences arterial oxygen tension and saturation, and occlusion of the PAVM increases saturation. When there is coexisting lung disease, ventilation-perfusion mismatch may also be a contributing factor [1].

\section{Case management and outcome} Following the diagnosis of a PAVM, the patient underwent percutaneous radiologically guided embolisation with improvement in his exercise tolerance to $800 \mathrm{~m}$. Postprocedure, his oxygen saturations on air were 97\% when sitting and 95\% when supine.

On closer questioning, he reported frequent nosebleeds over a number of years and was seen by the ear, nose and throat team who confirmed the rhinological appearances were compatible with hereditary haemorrhagic teleangiectasia (HHT).

Despite his age at presentation, the diagnosis had not been previously considered. Subsequently, his daughter and grandsons reported recurrent nose bleeds, and were advised to seek screening for HHT.

\section{Discussion}

PAVMs are direct communications between branches of the pulmonary artery and vein allowing blood to pass from one to the other without traversing the pulmonary capillary bed, resulting in the pulmonary arterial blood passing through these right-to-left shunts without being oxygenated. As the normal pulmonary capillary bed also prevents bacteria and emboli from reaching the systemic circulation, bypassing this system can potentially lead to both septic and embolic events.

PAVMs due to HHT account for at least 90\% of cases and these develop over time. $\mathrm{HHT}$ is an autosomal dominant disorder of the vasculature resulting in AVMs and telangiectasia. PAVMs in HHT may be present in childhood but grow and, in some, possibly many cases, develop de novo in puberty.

Acquired PAVMs are far less common and have been reported to occur most frequently following thoracic surgery for congenital valvular heart disease [2]. Lesions have been classified as simple (with one feeding artery), complex (with more than one feeding artery) and diffuse (rare but with hundreds of malformations) [3].

\section{Presentation}

The cardiorespiratory clinical picture depends on the size of the shunt and associated clinical parameters, such as concurrent cardiopulmonary disease and iron deficiency. Shunts over $20 \%$ of the cardiac output can lead to cyanosis, clubbing and polycythaemia. Large shunts commonly cause minimal or no clinical symptoms even when associated with clinical signs such as cyanosis, clubbing or polycythaemia. As the hypoxaemia is due to a right-to-left shunt, the cyanosis may only partially correct with oxygen therapy. More acute presentations are due to paradoxical emboli causing stroke, or due to rupture of the AVM with resultant massive pulmonary haemorrhage or haemothorax. A 2:1 female 
Table 2. Presenting features of pulmonary arteriovenous malformations

\section{Symptoms}

Asymptomatic

Breathlessness

Haemoptysis

Focal neurological deficit (stroke/brain abscess)

\section{Signs}

\section{Cyanosis}

Clubbing

Telangiectasia (if associated with HHT)

Polycythaemia

Pulmonary bruit (exacerbated by the Müller manoeuvre)

Focal neurological deficit (stroke/brain abscess) preponderance has been reported in older series.

As most cases are associated with $\mathrm{HHT}$, there may be a history of epistaxis or neurological symptoms (due to paradoxical emboli) but many PAVMs are asymptomatic.

Presenting features of PAVMs are shown in table 2.

\section{Complications of PAVMs}

PAVMs carry significant risks [4]. Neurological complications are most commonly reported (up to $59 \%$ of patients) and are due to paradoxical emboli via pulmonary circulation. The most common neurological manifestation is migraine but there is significant risk of stroke ( $1 \%$ per annum) and brain abscess ( $0.5 \%$ per annum) with the risk being unrelated to the size of the lesion [5]. Pulmonary complications are rare with one study showing an incidence of $8 \%$ in a group of patients referred for interventional radiology. These complications are more common in women, particularly when pregnant. Prevention of these complications is the main rationale for prophylactic treatment due to the potentially catastrophic nature of stroke and haemorrhage.

\section{Investigations}

Full history and examination is required to elicit any personal or family history of any symptomatology suggestive of HHT.

A chest radiograph may demonstrate a localised abnormality (as it did in this case) but contrast-enhanced CT is more accurate in diagnosis and determining the anatomy. Pulmonary function tests, pulse oximetry and demonstration of orthodeoxia all add to the overall clinical picture. A bubble contrast echocardiogram will demonstrate a right-to-left shunt but not identify the cause or the extent of the defect. Of note, bubble contrast echocardiography can be positive in control subjects. Pulmonary angiogram allows definition of the vasculature during embolisation [2] while CT angiogram has better resolution for small PAVMs.

Magnetic resonance angiography is an option for younger patients where the radiation risk is of greater concern but may not identify all clinically significant lesions.

\section{Treatment}

PAVMs were previously treated with surgical resection but now embolisation with coils and plugs is the treatment of choice. PAVMs should be embolised if technically feasible, which is generally possible with feeding arteries of $2 \mathrm{~mm}$ or more. Treatment of PAVMs by embolisation is a safe and effective way to reduce lifetime risk of embolic events and abscess formation as well as treating haemoptysis and improving oxygenation [6].

\section{Screening}

Following identification of a patient with a PAVM, consideration needs to be given to screening of family members due to the autosomal dominant inheritance of $\mathrm{HHT}$. The diagnostic criteria (Curacao criteria) for HHT are outlined in table 3. HHT can be definitively diagnosed when three or four of the criteria are met.

There is no clear consensus on the optimal screening tool. In some centres, the demonstration of a right-to-left shunt by contrast echocardiography has a reported sensitivity and negative predictive value $>95 \%$ [7]. The low specificity (62\%) may indicate microscopic PAVMs that are too small to be recognised on CT imaging. Other 
Table 3. Curacao diagnostic criteria for HHT

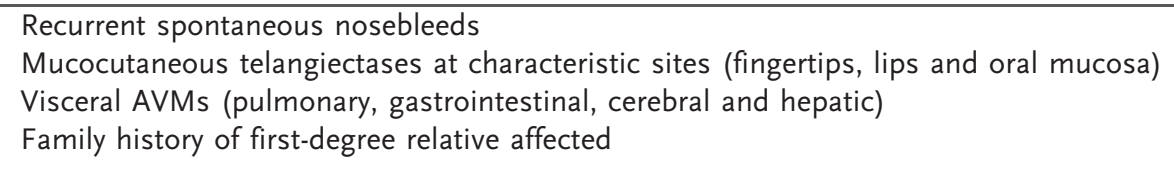

3-4 criteria: definite HHT; 2 criteria: possible HHT; o-1 criterion: unlikely HHT.

centres opt for chest radiography proceeding to contrast $\mathrm{CT}$ if any abnormality is detected and using contrast echocardiography if the chest radiograph is normal [8]. Genetic screening may of benefit to asymptomatic family members of a known HHT patient but, in the general patient population, the Curacao criteria are a more sensitive diagnostic tool.

\section{Special considerations: pregnancy and pulmonary hypertension}

PAVMs can become more problematic in pregnancy as the increase in cardiac output and blood volume can cause dilatation of the vasculature. The risk of PAVM rupture is difficult to quantify but is significantly higher in pregnancy. Therefore, women with known PAVMs need to be carefully counselled prepregnancy with emphasis on treatment prior to conceiving.

Overall maternal mortality in the UK is 4.67 per 100,000 maternities [9] but in women with PAVMs mortality is $1 \%$ with all deaths occurring in women without prepregnancy diagnosis of their PAVM [10]. Current UK recommendations [10] propose that, although the risk remains low and most pregnancies have no complications, the significant increase means that these patients should be managed as high-risk pregnancies. Therefore women with known PAVMs need to be carefully counselled pre-pregnancy and screened for PAVMs with emphasis on treatment prior to conceiving. Screening for cerebral AVMs should also be considered in those HHT patients with a family history of cerebral haemorrhage and, for those wanting regional anaesthesia, a spinal magnetic resonance imaging scan may be considered to exclude spinal AVMs. Antibiotic prophylaxis is required during delivery.
Patients with co-existing PAVMs and pulmonary hypertension can be difficult to manage, although the treatment options remain the same. Embolisation will preferentially correct areas with lower vascular resistance and paradoxically increases the risk of focally elevated pulmonary artery pressure with potentially disastrous consequences. In this group of patients, the risk of embolic events diminishes with the rise in pulmonary artery pressure and some patients fail to obtain symptomatic relief following embolisation. Therefore, it has been suggested that the risks of PAVM embolisation in patients with co-existing pulmonary hypertension may outweigh the benefits [6].

\section{Follow-up and patient education}

Patients with PAVMs require long-term follow-up. While embolisation may cure the malformation, up to $60 \%$ of patients have evidence of residual disease due to PAVMs too small to treat. Residual defects may also increase in size following embolisation of the larger vessels. Additionally, there is a risk of recanalisation over time of up to $20 \%$, either through the embolic device or from the development of collateral feeding arteries. Following treatment, patients will be regularly followed up but with urgent review and further CT assessment if their condition changes. Patients need to be given education regarding antibiotic prophylaxis for routine medical and dental procedures, the need for air filters on intravenous infusions, and the risks of decompression sickness in scuba diving [12].

As most PAVMs are associated with $\mathrm{HHT}$, a thorough assessment of the patient in this regard is required and familial screening may be indicated. 


\section{Further reading}

Cheng TO. Platypnoeaorthodeoxia syndrome: etiology, differential diagnosis and management. Cathet Cardiovasc Interv 1999; 47: 64-66. A discussion of the mechanism of orthodeoxia in cardiac disease

Hoeppner VH.

Orthocyanosis: a sign of pulmonary arteriovenous malformation. Thorax

1982; 37: 952-953.

\section{References}

1. Shovlin CL, Wilmshurst P, Jackson JE. Pulmonary arteriovenous malformations and other pulmonary aspects of HHT. Eur Respir Monogr 2011; 54: 218-245.

2. Khurshid I, Downie GH. Pulmonary arteriovenous malformation. Postgrad Med J 2002; 78: 191.

3. White RI, Pollak JS, Wirth JA. Pulmonary arteriovenous malformations: diagnosis and transcatheter embolotheraphy. J Vasc Interv Radiol 1996; 7: 787-804.

4. Cottin V, Dupuis-Girod S, Lesca G, et al. Pulmonary vascular manifestations of hereditary hemorrhagic telangiectasia (Rendu-Osler disease). Respiration 2007; 74: 361-378.

5. Shovlin CL, Jackson JE, Bamford KB, et al. Primary determinants of ischaemic stroke/brain abscess risks rare independent of severity of pulmonary arterovenous malformations in hereditary haemorrhagic telangiectasia. Thorax 2008; 63: 259-266.

6. Shovlin CL, Gibbs JSR, Jackson JE. Management of pulmonary arteriovenous malformations in pulmonary hypertensive patients: a pressure to embolise? Eur Respir Rev 2009; 18: 4-6.

7. Van Gent MW, Post MC, Luermans JG, et al. Screening for pulmonary arteriovenous malformations using transthoracic contrast echocardiography: a prospective study. Eur Respir J 2009; 33: 85-91.

8. Morrell NW. Screening for pulmonary arteriovenous malformations. Am J Respir Crit Care Med 2004; 169: 978-979.

9. Cantwell R, Clutton-Brock T, Cooper G, et al. Saving Mothers' Lives: reviewing maternal deaths to make motherhood safer: 2006-08. The Eighth Report on Confidential Enquiries into Maternal Deaths in the United Kingdom. BJOG 2011; 118: Suppl. 1, 1-203.

10. Shovlin CL, Sodhi V, McCarthy A, et al. Estimates of maternal risks of pregnancy for women with hereditary haemorrhagic telangiectasia (Osler-Weber-Rendu synfrome): suggested approach for obstetric services. BJOG 2008; 115: 1108-1115.

11. Olitsky SE. Hereditary hemorrhagic telangiectasia: diagnosis and management. Am Fam Phys 2010; 82: 785-790.

12. Gossage JR, Kanj G. Pulmonary arteriovenous malformations: a state of the art review. Am J Respir Crit Care Med 1998; 158: 643-661.

13. Shovlin CL, Guttmacher AE, Buscarini E, et al. Diagnostic criteria for hereditary hemorrhagic telangiectasia (Rendu-Osler-Weber syndrome). Am J Med Genet 2000; 91: 66-67. 